\title{
ON STRUCTURES OF INFINITE MODULES
}

\author{
BY \\ R. E. JOHNSON
}

Much of the literature on the structures of modules applies to those modules which possess a finite basis. The present paper is the development of a structure theory for particular infinite modules with countable bases. Generality of results is not as much the aim of the paper as is the application to problems concerning infinite matrices.

For a commutative field $P, \Xi$ is assumed to be a universal $P$-module which has a countable $P$-basis. A principal ideal ring $Q$ which contains $P$ is considered as an operator domain of $\Xi$. Then the main topic studied is under what conditions submodules of $\Xi$ have proper $Q$-bases.

In the first place, a complete characterization is given for the proper $Q$-bases of any $Q$-submodule of $\Xi$. This is represented as an infinite matrix, and is called the characteristic matrix of the submodule.

The finite case is studied in the third section. The results obtained are comparable with those of Ingraham and Wolf [3](1) and Chevalley [1]. The principal theorem is that every $Q$-module which possesses a finite $Q$-basis has a proper $Q$-basis.

The concepts of primitivity-defined somewhat as Chevalley defines it and index play an important role in determining conditions for a $Q$-module to have a proper $Q$-basis. In order to find these conditions, the non-regular elements $H$ of $\Xi$ are split from $\Xi$. The resulting $Q$-module $\Xi / H$ is regular. Then necessary and sufficient conditions are found for both $H$ and $\Xi / H$ to have a proper $Q$-basis.

If the operator domain of $\Xi$ be considered as $Q /(m), m$ not a unit of $Q$, then in the fifth section it is seen that $\Xi$ possesses a proper $Q /(m)$-basis.

As an application of these results, $\Xi$ is taken to be the set of all vectors over $P$ of order type $\omega$ which are finitely nonzero. The total operator domain of $\Xi$ is a certain ring of infinite matrices, $\mathfrak{M}_{\omega}$. Then any element $A$ of $\mathfrak{M}_{\omega}$ can be transformed into a direct sum of finite matrices only if $\Xi$ has a proper $P[A]$-basis.

The algebraic theory assumed herein can be found in almost any book on modern algebra-specific attention is called to MacDuffee [4] and Zassenhaus [5].

1. Introduction. Let $P$ denote a commutative field, and $Q$ a principal ideal

Presented to the Society, April 18, 1942; received by the editors April 28, 1942, and, in revised form, October 13, 1942.

(1) The numbers in brackets refer to the bibliography at the end of the paper. 
ring which contains $P\left({ }^{2}\right)$. The universal $P$-module (linear set over $P$ ) of all modules discussed below will be labelled by $\Xi$. It is assumed to have $Q$ as an operator domain. While $\Xi$ will in general have an infinite number of elements, yet only finite sums are ever considered. Small Greek letters will always denote elements of $\Xi$, capital Greek letters will stand for subsets of $\Xi$, and small Latin letters will be used for elements of $Q$.

For subsets $\Xi_{1}, \Xi_{2}, \ldots$ of $\Xi, \Xi_{1} \vee \Xi_{2} \vee \ldots$ is used to denote the least $P$-module in $\Xi$ which contains all $\Xi_{i}$, while $\Xi_{1} \wedge \Xi_{2} \wedge \ldots$ denotes the settheoretic intersection of all $\Xi_{i}$. If the $\Xi_{i}, i=1,2, \cdots$, are $P$-modules, and $\Xi^{\prime}=\Xi_{1} \vee \Xi_{2} \vee \cdots$, then $\Xi^{\prime}$ is the supplementary sum of the $\Xi_{i}$, written

$$
\Xi^{\prime}=\Xi_{1}+\Xi_{2}+\cdots
$$

in case the representation of every element of $\Xi^{\prime}$ by sums of elements in the $\Xi_{i}$ is unique. This is equivalent to the condition

$$
\Xi_{k} \wedge\left(\Xi_{1} \vee \Xi_{2} \vee \cdots \vee \Xi_{k-1}\right)=0, \quad k=2,3, \cdots .
$$

For $\Xi_{2} \subset \Xi_{1}, \Xi_{1}-\Xi_{2}$ is the set of all elements of $\Xi_{1}$ not in $\Xi_{2}$.

The universal $P$-module $\Xi$ is said to have the $P$-basis $\left(\xi_{1}, \xi_{2}, \cdots\right)$, finite or infinite, if

$$
\Xi=P\left[\xi_{1}, \xi_{2}, \cdots\right],
$$

this last being the set of all finite combinations $\sum a_{i} \xi_{i}, a_{i} \in P$. If the set $\left(\xi_{1}, \xi_{2}, \cdots\right)$ is $P$-linearly independent-that is, for every finite $\operatorname{sum} \sum a_{i} \xi_{i}=0$, all $a_{i}=0$ - this basis is called regular. In this case one has

$$
\Xi=P \xi_{1}+P \xi_{2}+\cdots, P \xi_{i}=P\left[\xi_{i}\right] .
$$

The following axiom is assumed throughout the paper.

Fundamental Axiom. The universal module $\Xi$ has a countable P-basis.

A consequence of this axiom (see Ingraham [2]) is

Theorem 1.1. Every $P$-submodule $\Xi_{1}$ of $\Xi$ has a proper P-basis.

The set $\left(\xi_{1}, \xi_{2}, \ldots\right)$ is a $Q$-basis for $\Xi_{1}$ in case $\boldsymbol{\Xi}_{1}=Q\left[\xi_{1}, \xi_{2}, \ldots\right]$. The set $\Xi_{1}$ is a $Q$-module in case $Q \xi \subset \Xi_{1}$ for every $\xi \in \Xi_{1}$.

Definition 1.2. A set of elements $\left(\xi_{1}, \xi_{2}, \cdots\right)$ is a proper Q-basis of $\Xi_{1}$, and is thus $Q$-linearly independent, if and only if

$$
\xi_{i} \neq 0 \text {, }
$$$$
i=1,2, \cdots,
$$

and

$$
\Xi_{1}=Q \xi_{1}+Q \xi_{2}+\cdots
$$

(2) As a particular instance of these concepts, we can consider $P$ as the rational field and $Q$ the ring $P[x]$ of all polynomials in the indeterminate $x$ with coefficients in $P$. The first part of $\S 6$ might well be read first to give one a concrete example of the sets $Q$ and $\Xi$. 
An element $a$ of $Q$ is an annihilator of $\xi$ if $a \xi=0$. If $a_{1}$ and $a_{2}$ are two annihilators of $\xi$, then $b_{1} a_{1}+b_{2} a_{2}$ is an annihilator of $\xi$ for any two elements $b_{1}$ and $b_{2}$ of $Q$. Thus the set of all annihilators of $\xi$ form an ideal. Since $Q$ is by assumption a principal ideal ring, this ideal is principal of the form $(h)$. As $h$ divides all annihilators of $\xi$, it will be called a minimum annihilator of $\xi$. It is unique up to a unit factor.

Definition 1.3. If $a \xi=0$ implies $a=0$, then $\xi$ is called regular. If every nonzero element of a $Q$-module $\Xi_{1}$ is regular, then $\Xi_{1}$ is called regular.

If $\xi$ and $\eta$ are two non-regular elements of $\Xi$ with minimum annihilators $a_{1}$ and $a_{2}$, respectively, then for any elements $b_{1}$ and $b_{2}$ of $Q, b_{1} \xi+b_{2} \eta$ is annihilated by $a_{1} a_{2}$. Thus the set of all non-regular elements of $\Xi$ forms a $Q$-module which we shall label $H$. Suppose $\left(\eta_{1}, \eta_{2}, \cdots\right)$ is a $P$-basis for $H$, with $h_{i}$ the minimum annihilator of $\eta_{i}$. Then we have defined a set $\left(p_{1}, p_{2}, \cdots\right)$ of primes of $Q$, which are all the distinct prime factors of the $h_{i}, i=1,2, \cdots$ ( $p$ and $q$ are not distinct if $p=q c, c$ a unit). Now any element $\eta$ of $H$ is of the form $\eta=\sum_{i=1}^{n} a_{i} \eta_{i}, a_{i} \in P$, so $\eta$ is annihilated by some product of the primes $p_{j}$. For any element $p$ of $Q$, let $H_{p}$ be the set of all elements of $H$ annihilated by some power of $p$. Then $H_{p}$ is a $Q$-module.

THEOREM 1.4. $H=H_{p_{1}}+H_{p_{2}}+\cdots$.

To prove this, let $\eta$ be any element of $H$, and let $\prod_{i=1}^{n} p_{i}^{t_{i}}$ be its minimum annihilator. Then there exist elements $s_{i}$ such that

If

$$
\sum_{j=1}^{n} s_{j} \prod_{i=1, i \neq j}^{n} p_{i}^{t_{i}}=1
$$

$$
\eta_{j}=s_{j} \prod_{i=1, i \neq j}^{n} p_{i}^{t_{i}}
$$

then $\eta_{i} \in H_{p_{i}}, \eta=\eta_{1}+\eta_{2}+\cdots+\eta_{n}$.

As an immediate consequence of this theorem, we have that for any $Q$-module $H_{1} \subset H$,

$$
H_{1}=H_{1} \wedge H_{p_{1}}+H_{1} \wedge H_{p_{2}}+\cdots \text {. }
$$

Any $P$-module $\Xi_{1}$ is fundamentally an abelian group, so that the quotient group $\Xi_{1} / \Xi_{2}$ is well defined for any $P$-submodule $\Xi_{2}$ of $\Xi_{1}$, and is itself a $P$-module. Likewise, if $\Xi_{1}$ and $\Xi_{2}$ are $Q$-modules with $\Xi_{2} \subset \Xi_{1}$, then $\Xi_{1} / \Xi_{2}$ is a $Q$-module.

If $H_{1}$ is the set of all non-regular elements of the $Q$-module $\Xi_{1}$, then $\Xi_{1} / H_{1}$ has elements of the form $\left(\xi+H_{1}\right)$ (this should not be confused with the supplementary sum-it means the set of all elements of the form $\xi+\eta, \eta \in H_{1}$ ) for $\xi \in \Xi_{1}$. Suppose $a\left(\xi+H_{1}\right)=\left(H_{1}\right)$ for some element $a \in Q, \xi \in \Xi_{1}-H_{1}$. Then $a \xi \in H_{1}$ so that $a=0$. Thus $\Xi_{1} / H_{1}$ is a regular $Q$-module. 
2. Invariants of proper $Q$-bases. Let $\Xi_{1}$, a $Q$-module, have a proper $Q$-basis $\left(\eta_{1}, \eta_{2}, \cdots, \xi_{1}, \xi_{2}, \cdots\right)$, the $\xi_{i}$ being regular elements, and $\eta_{i}$ having minimum annihilator $q_{i}, i=1,2, \cdots$. If $H_{1}=Q\left[\eta_{1}, \eta_{2}, \cdots\right], \Omega_{1}=Q\left[\xi_{1}, \xi_{2}, \cdots\right]$, then $\Xi_{1}=H_{1}+\Omega_{1}, H_{1} \subset H$.

Definition 2.1. The infinite matrix $\left(n_{r s} ; r=0,1,2, \cdots ; s=1,2, \cdots\right)$, in which $n_{r s}$ is the number of the $q_{j}, j=1,2, \cdots$, divisible by $p_{r}^{s}$ but not by $p_{r}^{s+1}$ for $r, s=1,2, \cdots$, and $n_{0 s}$ is the cardinal number of the set $\left(\xi_{1}, \xi_{2}, \cdots\right)$, $s=1,2, \cdots$, is called the characteristic matrix $\left(^{3}\right)$ of the proper Q-basis $\left(\eta_{1}, \eta_{2}, \cdots, \xi_{1}, \xi_{2}, \cdots\right)$. The elements of this matrix are integers or $\boldsymbol{\aleph}_{0}$.

ThEOREM 2.2. The characteristic matrix is an invariant of the class of all proper Q-bases of $\boldsymbol{\Xi}_{1}$.

To prove this, let $\left(\eta_{j 1}, \eta_{j 2}, \cdots, \xi_{j 1}, \xi_{j 2}, \cdots\right), j=1,2$, be two proper $Q$-bases of $\Xi_{1}$, with the $\xi_{j i}$ regular and $\eta_{j i}$ annihilated minimally by $q_{j i}$, $i=1,2, \cdots$. The number of elements of the set $\left(q_{j 1}, q_{j 2}, \cdots\right)$ divisible by $p_{r}^{s}$ but not by $p_{r}^{s+1}$ is denoted by $n_{j r s}, r, s=1,2, \ldots$. The cardinal number of the set $\left(\xi_{j 1}, \xi_{j 2}, \cdots\right)$ is denoted by $n_{j 08}$, so that $n_{j 01}=n_{j 02}=\cdots$. We shall first show that $n_{111}=n_{211}$.

Select $r_{i j}$ from $Q$ so that $q_{i j}=p_{1}^{i_{i j}} r_{i j}$ with the greatest common divisor of $p_{1}$ and $r_{i j}$, denoted $\left(p_{1}, r_{i j}\right)$, equal to 1 . The nonzero elements of the set $\left(r_{j 1} \eta_{j 1}, r_{j 2} \eta_{j 2}, \cdots\right)$ are $Q$-linearly independent: let us relabel these nonzero elements $\left(\alpha_{j 1}, \alpha_{j 2}, \cdots\right)$. Then

$$
Q \alpha_{11}+Q \alpha_{12}+\cdots=Q \alpha_{21}+Q \alpha_{22}+\cdots,
$$

this being the set $H_{p_{1}}$ of all elements of $H_{1}$ annihilated by some power of $p_{1}$.

If $n_{111}=n_{211}=\aleph_{0}$, the first step of the proof is concluded. Thus assume $n_{111}=n<\boldsymbol{N}_{0}$. Let us separate the $\alpha_{j i}$ in to the sets $\left(\beta_{j 1}, \beta_{j 2}, \cdots\right),\left(\gamma_{j 1}, \gamma_{j 2}, \cdots\right)$ with the first set being all the $\alpha_{j i}$ annihilated by $p_{1}$, and the second set the remainder of the $\alpha_{j i}$. It is observed that no member of the set $\left(\beta_{11}, \beta_{12}, \cdots, \beta_{1 n}\right)$ could be in the module $Q\left[\gamma_{21}, \gamma_{22}, \cdots\right]$. For if

$$
\beta_{11}=\sum_{i=1}^{t} c_{i} \gamma_{2 i}
$$

then each $c_{i}$ must be divisible by $p_{1}$ as $p_{1}$ annihilates $\beta_{11}$, but does not annihilate any $\gamma_{j i}$. Thus $\beta_{11}=p_{1} \gamma$. However, $\gamma \in Q\left[\beta_{11}, \beta_{12}, \cdots, \gamma_{11}, \gamma_{12}, \cdots\right]$, say

$$
\gamma=\sum_{i=1}^{s} d_{i} \beta_{1 i}+\sum_{i=1}^{r} e_{i} \gamma_{1 i}
$$

so that $\beta_{11}=\sum_{i=1}^{r} p_{1} e_{i} \gamma_{1 i}$, which is impossible. From (1), we have

(3) This bears no relationship with the ordinary concept of characteristic matrix. The name was chosen because of the connection between this matrix and the characteristic divisors of certain infinite matrices. 


$$
\begin{array}{ll}
\beta_{1 i}=\sum_{j=1}^{m_{i}} b_{1 i} \beta_{2 j}+p_{1} \delta_{1 i}, & \delta_{1 i} \in Q\left[\gamma_{21}, \gamma_{22}, \cdots\right], \\
\beta_{2 i}=\sum_{j=1}^{n} b_{2 i} \beta_{1 j}+p_{1} \delta_{2 i}, & \delta_{2 i} \in Q\left[\gamma_{11}, \gamma_{12}, \cdots\right] .
\end{array}
$$

A substitution yields, if we let $m$ be the maximum $m_{i}$, and $b_{1 i j}=0, m_{i}<j \leqq m$,

$$
\begin{aligned}
& \beta_{1 i}=\sum_{j=1}^{m} \sum_{k=1}^{n} b_{1 i j} b_{2 j k} \beta_{1 k}+p_{1} \delta_{1}, \\
& \beta_{2 i}=\sum_{j=1}^{n} \sum_{k=1}^{m} b_{2 i} b_{1 j k} \beta_{2 k}+p_{1} \delta_{2} .
\end{aligned}
$$

From the $Q$-linear independence of the sets $\left(\beta_{j 1}, \beta_{j 2}, \cdots, \gamma_{j 1}, \gamma_{j 2}, \cdots\right)$ one must have $p_{1} \delta_{1}=p_{1} \delta_{2}=0$. If the matrices $B_{1}$ and $B_{2}$ are defined as

$$
\begin{aligned}
& B_{1}=\left(b_{1 r s} ; r=1, \cdots, n ; s=1, \cdots, m\right), \\
& B_{2}=\left(b_{2 r s} ; r=1, \cdots, m ; s=1, \cdots, n\right),
\end{aligned}
$$

then from (2) one can conclude (using $I_{k}$ as the unit matrix of order $k^{2}$ )

$$
B_{1} B_{2}=I_{n}, \quad B_{2} B_{1}=I_{m} .
$$

However $B_{1}$ and $B_{2}$ have elements in the field $Q /\left(p_{1}\right)$, so that $m$ and $n$ must be equal. Also, from (2), for $t>n$,

$$
\beta_{2 t}=\sum_{j=1}^{n} \sum_{k=1}^{m} b_{2 i j} b_{1 j k} \beta_{2 k}
$$

which is impossible, as the set $\left(\beta_{21}, \beta_{22}, \cdots\right)$ is $Q$-linearly independent. The conclusion is that there are $n$ elements in the set $\left(\beta_{21}, \beta_{22}, \cdots\right)$, so that $n_{111}=n_{211}$.

In order to show that $n_{11 t}=n_{21 t}$, consider the set $p_{1}^{t-1} H_{p_{1}}$, which has the two proper (if we exclude the zero elements) $Q$-bases $\left(p_{1}^{t-1} \alpha_{j 1}, p_{1}^{t-1} \alpha_{j 2}, \cdots\right)$, $j=1$, 2. From the paragraph above, the number of nonzero elements annihilated by $p_{1}$ in each basis is the same. This number must be the number of $q_{j i}$ divisible exactly by $p_{1}^{t}$-so that $n_{11 t}=n_{21 t}$.

We need only to show that $n_{101}=n_{201}$ to complete the proof. Let

$$
\begin{array}{ll}
\xi_{1 j}=\sum_{k} b_{1 j k} \eta_{2 k}+\sum_{k=1}^{m_{1 j}} d_{1 j k} \xi_{2 k}, & j=1,2, \cdots, n_{101}, \\
\xi_{2 j}=\sum_{k} b_{2 j k} \eta_{1 k}+\sum_{k=1}^{m_{2 j}} d_{2 j k} \xi_{1 k}, & j=1,2, \cdots, n_{201} .
\end{array}
$$

Case 1. Let $n_{101}=N_{0}, n_{201}$ finite: if $m$ be the maximum $m_{2 j}, j=1,2, \cdots, n_{201}$, then $\Xi_{1} \subset Q\left[\eta_{11}, \eta_{12}, \cdots, \xi_{11}, \xi_{12}, \cdots, \xi_{1 m}\right]$ which is impossible. 

that

Case 2. Let $n_{101}, n_{201}$ both be finite: then there exists an element $c \in Q$ such

$$
\begin{aligned}
c \xi_{1 j}=c \sum_{i=1}^{n_{201}} d_{1 j i} \xi_{2 i}, & j=1,2, \cdots, n_{101}, \\
c \xi_{2 j}=c \sum_{i=1}^{n_{101}} d_{2 j i} \xi_{1 i}, & i=1,2, \cdots, n_{201} .
\end{aligned}
$$

If $D_{k}=\left(d_{k r s}\right), k=1,2$, then by the method above

$$
c D_{1} D_{2}=c I_{n_{101}}, \quad c D_{2} D_{1}=c I_{n_{201}} \text {. }
$$

As $Q$ can always be imbedded in a field, we can consider $D_{1}$ and $D_{2}$ as having elements in a field, so that $n_{101}=n_{201}$. Thus the characteristic matrices of the two bases must be equal.

Consider again the proper $Q$-basis for $\Xi_{1}$ given at the beginning of this section. If $q_{1}=c p_{i_{1}}^{t_{1}} \cdots p_{n_{n}}^{t_{n}}, c$ a unit and all $t_{i} \geqq 1$, then let

$$
\eta_{11}=p_{i_{2}}^{t_{2}} \cdots p_{i_{n}}^{t_{n}} \eta_{1}, \quad \eta_{12}=p_{i_{1}}^{t_{1}} p_{i_{3}}^{t_{3}} \cdots p_{i_{n} \eta_{1}}^{t_{n}}, \cdots, \quad \eta_{1 n}=p_{i_{1}}^{t_{1}} \cdots p_{i_{n-1} \eta_{1}}^{t_{n-1}}
$$

We see that $Q \eta_{1}=Q \eta_{11}+Q \eta_{12}+\cdots+Q \eta_{1 n}$ where each $\eta_{1 i}$ is minimally annihilated by a power of a prime. Now this can be done for every $\eta_{i}$, so it is apparent that $\Xi_{1}$ has a proper $Q$-basis $\left(\beta_{1}, \beta_{2}, \cdots, \xi_{1}, \xi_{2}, \cdots\right)$, with the $\xi_{i}$ regular and the $\beta_{i}$ possessing powers of primes as minimum annihilators.

With the use of this basis, the completeness of the invariant characteristic matrix $\left(n_{r s}\right)$ will be shown by the following

ThEOREM 2.3. For any set $\left(r_{1}, r_{2}, \cdots\right)$ of elements of $Q$ such that the number of these elements divisible by $p_{r}^{s}$ but not by $p_{r}^{s+1}$ is $n_{r s}$, there exists a proper $Q$-basis $\left(\alpha_{1}, \alpha_{2}, \cdots, \xi_{1}, \xi_{2}, \cdots\right)$ of $\Xi_{1}$, with $r_{j}$ the minimum annihilator of $\alpha_{j}$, and $n_{01}$ the number of elements in the set $\left(\xi_{1}, \xi_{2}, \cdots\right)$.

If $r_{1}=c p_{i_{1}}^{t_{1}} \cdots p_{t_{k}}^{t_{k}}$, all $t_{i}>0$ and $c$ a unit, then let $j_{1}$ be the minimum integer such that $\beta_{j_{1}}$ has minimum annihilator $p_{i_{1}}^{t_{1}}$, and, in general, let $j_{s}$ be the minimum integer such that $\beta_{j_{s}}$ has minimum annihilator $p_{i_{s}}^{i_{s}}$. Now define $\alpha_{1}=\beta_{j_{1}}+\beta_{j_{2}}+\cdots+\beta_{j_{k}}$, so that $\alpha_{1}$ has minimum annihilator $\gamma_{1}$. Discard $\beta_{j_{1}}, \beta_{j_{2}}, \cdots, \beta_{j_{k}}$ from the set $\left(\beta_{1}, \beta_{2}, \cdots\right)$, and with the remaining set carry through a similar process for $r_{2}$, obtaining $\alpha_{2}$. From Theorem 2.2, there will be precisely enough elements in the set $\left(\beta_{1}, \beta_{2}, \cdots\right)$ to carry this process to completion, using all the $r_{i}^{\prime s}$. We can use the same regular elements in the new basis as in the old one. This completes the proof.

COROLlARY 2.4. If all the elemenis of $H_{p}$ are annihilated by some power of the prime $p$, and $H_{p}$ has a proper $Q$-basis, then the number of elements in any proper $Q$-basis of $H_{p}$ is an invariant of $H_{p}$. 
If a proper $Q$-basis has an infinite number of elements, obviously any $Q$-basis has an infinite number of elements. Suppose $\left(\eta_{1}, \cdots, \eta_{n}\right)$ is a proper $Q$-basis of $H_{p}$, and $\left(\alpha_{1}, \cdots, \alpha_{m}\right)$ any $Q$-basis. Then

so that

$$
\eta_{i}=\sum_{j=1}^{m} a_{i j} \alpha_{j}, \quad \alpha_{j}=\sum_{k=1}^{n} b_{j k} \eta_{k},
$$

$$
\begin{aligned}
\sum_{j=1}^{m} a_{i j} b_{j k} & \equiv 1 \bmod p \text { if } j=k, \\
& \equiv 0 \bmod p \text { otherwise, }
\end{aligned}
$$

which is possible only if $m \geqq n$. This leads to

THEOREM 2.5. If $H_{p}$ has a proper $Q$-basis, then the number of elements in any $Q$-basis cannot be less than the number of elements in a proper Q-basis.

3. The finite case. Before the general case is studied, it is necessary to consider those submodules of $\Xi$ which have finite $Q$-bases.

LEMma 3.1. If $\Xi_{1}$ has a finite $Q$-basis, and $\Xi_{2} \subset \Xi_{1}$, 寻 a $Q$-module, then $\Xi_{2}$ has a finite Q-basis. The Q-basis for $\Xi_{2}$ can be chosen so as not to have more elements than the given $Q$-basis for $\Xi_{1}$.

To prove this, let $\left(\xi_{1}, \xi_{2}, \cdots, \xi_{n}\right)$ be a $Q$-basis of $\Xi_{1}$. For any $m \leqq n$, there exists a maximum ideal $\mathfrak{a}_{m} \subset Q$ such that

$$
\mathfrak{a}_{m} \xi_{m} \equiv 0 \bmod \Xi_{2} \vee Q\left[\xi_{1}, \xi_{2}, \cdots, \xi_{m-1}\right] .
$$

As $Q$ is a principal ideal ring, $\mathfrak{a}_{m}=\left(s_{m}\right)$. Select $\eta_{m} \in \Xi_{2}$ so that

$$
\eta_{m} \equiv s_{m} \xi_{m} \bmod Q\left[\xi_{1}, \xi_{2}, \cdots, \xi_{m-1}\right], \quad \eta_{m}=0 \text { if } s_{m}=0 .
$$

Suppose $\eta \in \Xi_{2}$, so that $\eta=\sum_{i=1}^{k} a_{i} \xi_{i}$ with $a_{k} \xi_{k} \neq 0$. Then

$$
a_{k} \xi_{k} \equiv \eta \bmod Q\left[\xi_{1}, \xi_{2}, \cdots, \xi_{k-1}\right]
$$

which implies

$$
\eta \equiv b_{k} \eta_{k} \bmod Q\left[\xi_{1}, \xi_{2}, \cdots, \xi_{k-1}\right] .
$$

Thus $\eta-b_{k} \eta_{k} \in Q\left[\xi_{1}, \xi_{2}, \cdots, \xi_{k-1}\right]$, and by induction $\eta=\sum_{i=1}^{k} b_{i} \eta_{i}$. This shows that $\left(\eta_{1}, \eta_{2}, \cdots, \eta_{n}\right)$ is a $Q$-basis for $\Xi_{2}$, and establishes the lemma.

If $\left(\xi_{1}, \xi_{2}, \cdots, \xi_{n}\right)$ is a $P$-basis for the $Q$-module $\Xi_{1}$, then for any element $\xi \in \Xi_{1}$ and any element $b \in Q, b$ not a unit, the set $\left(\xi, b \xi, \cdots, b^{n} \xi\right)$ must be $P$-linearly dependent. Thus $\sum_{i=1}^{n} a_{i} b^{i} \xi=0$ for some elements $a_{i} \in P$; we have

LemmA 3.2. If $\Xi_{1}$ is a $Q$-module with a finite $P$-basis, then $\Xi_{1} \subset H$.

LEMmA 3.3. If $\Xi_{1}$ is a $Q$-module with a finite $Q$-basis, and $\Xi_{1} \subset H$, then $\boldsymbol{\Xi}_{1}$ has a proper Q-basis. 
To prove this, let $\Xi_{1}=Q\left[\xi_{1}, \xi_{2}, \cdots, \xi_{n}\right]$ with $\xi_{i}$ annihilated minimally by $r_{i}$, so that $\prod_{i=1}^{n} r_{i}$ annihilates $\Xi_{1}$. Let $\left(p_{1}, p_{2}, \cdots, p_{m}\right)$ be all the distinct prime factors of the $r_{i}, i=1,2, \cdots, n$. If $H_{i}$ denotes the set of all elements of $\Xi_{1}$ annihilated by some power of $p_{i}$, then from Theorem 1.4,

$$
\Xi_{1}=H_{1}+H_{2}+\cdots+H_{m} \text {. }
$$

We will prove that each $H_{i}$ has a proper $Q$-basis, which will imply a proper $Q$-basis for $\Xi_{1}$.

Denote by $t_{1}$ the minimum integer such that $p_{1}^{t_{1}} H_{1}=0$. Then there exists an element $\eta_{1} \in H_{1}$ for which $p_{1}^{i_{1}}$ is the minimum annihilator. Recursively, if $t_{k}$ is the minimum integer such that $p_{1}^{t_{k}} H_{1} \equiv 0 \bmod Q\left[\eta_{1}, \eta_{2}, \cdots, \eta_{k-1}\right]$, then there exists an element $\eta_{k} \in H_{1}$ which has $p_{1}^{t_{k}}$ as minimum annihilator $\bmod Q\left[\eta_{1}, \eta_{2}, \cdots, \eta_{k-1}\right]$. It follows that $t_{1} \geqq t_{2} \geqq \cdots \geqq t_{k}$.

Assume that the set $\left(\eta_{1}, \eta_{2}, \cdots, \eta_{k-1}\right)$ is $Q$-linearly independent, and that $p_{1}^{t_{k}} \eta_{k} \neq 0$. From above, we must have

$$
p_{1}^{t_{k}} \eta_{k}=\sum_{i=1}^{k-1} a_{i} \eta_{i}
$$

If this equation be multiplied by the $\left(t_{k-1}-t_{k}\right)$ th power of $p_{1}$, it is apparent that $p_{1}^{z_{k}} \mid a_{k-1}\left(p_{1}^{z_{k}}\right.$ divides $\left.a_{k-1}\right)$. Similarly, it can be verified that $p_{1}^{t_{k}} \mid a_{i}$, $i=1,2, \cdots, k-1$, so that $a_{i}=b_{i} p_{1}^{k_{k}}$. Then if

$$
\bar{\eta}_{k}=\eta_{k}-\sum_{i=1}^{k-1} b_{i} \eta_{i}
$$

the set $\left(\eta_{1}, \eta_{2}, \cdots, \eta_{k-1}, \bar{\eta}_{k}\right)$ is $Q$-linearly independent. If this process were not finite, we would have a submodule of $\Xi_{1}$ containing an infinite proper $Q$-basis. This is not possible in view of Theorem 2.5 and Lemma 3.1.

THEOREM 3.4. If $\Xi_{1}$ is a Q-module with a finite Q-basis, then $\Xi_{1}$ has a proper Q-basis.

This follows from Lemma 3.3 if all the elements of $\Xi_{1}$ are non-regular. Thus let $H_{1}=\Xi_{1} \wedge H \neq 0$, so that $\Xi_{1} / H_{1}$ is regular. As $\Xi_{1}$ has a finite $Q$-basis, so must $\Xi_{1} / H_{1}$ : denote this basis by $\left(\xi_{1}, \xi_{2}, \cdots, \xi_{n}\right)$, all $\xi_{i} \neq 0$.

Assume every $Q$-module contained in $\Xi_{1} / H_{1}$ which is generated by $k$ or fewer elements has a proper (finite) $Q$-basis. Also assume that the set $\left(\xi_{1}, \xi_{2}, \cdots, \xi_{k}\right)$ is $Q$-linearly independent, and that $\xi_{k+1}$ has a nonzero minimum annihilator $q_{k+1} \bmod Q\left[\xi_{1}, \xi_{2}, \cdots, \xi_{k}\right]$. Then there exist elements $q_{1}, \cdots, q_{k} \in Q$ such that

$$
\sum_{i=1}^{k+1} q_{i} \xi_{i}=0, \quad\left(q_{1}, q_{2}, \cdots, q_{k+1}\right)=1 .
$$

In the case under consideration, it is well known (see MacDuffee [4, 
p. 227]) that there exists a matrix $C=\left(c_{r s}\right)$ of $(k+1)^{2}$ elements with $c_{k+1 j}=q_{j}$, $j=1,2, \cdots, k+1$, such that $|C|=1$ and $C$ has a unique inverse $B=\left(b_{r s}\right)$.

Let .

$$
\alpha_{i}=\sum_{j=1}^{k+1} c_{i j} \xi_{j}, \quad i=1,2, \cdots, k .
$$

Remembering that $\sum_{t=1}^{k+1} c_{k+1 i} \xi_{i}=0$, we see that

$$
\sum_{j=1}^{k} b_{i j} \alpha_{j}=\sum_{s, t=1}^{k+1} b_{i s} c_{s t} \xi_{t}=\xi_{i}, \quad i=1,2, \cdots, k+1 .
$$

Thus $Q\left[\alpha_{1}, \alpha_{2}, \cdots, \alpha_{k}\right]=Q\left[\xi_{1}, \xi_{2}, \cdots, \xi_{k+1}\right]$. By assumption, this set has a proper $Q$-basis. An obvious induction leads to a proper $Q$-basis for $\Xi_{1} / H_{1}$. If this basis is $\left(\beta_{1}+H_{1}, \beta_{2}+H_{1}, \cdots, \beta_{t}+H_{1}\right)$, then

$$
\Xi_{1}=H_{1}+Q \beta_{1}+Q \beta_{2}+\cdots+Q \beta_{t},
$$

and the theorem follows.

4. The general case. We now turn to the consideration of any $Q$-submodule of $\Xi$, and develop conditions under which it possesses a proper Q-basis.

DeFINITION 4.1. For the Q-modules $\Xi_{1}, \Xi_{2}$ with $\Xi_{2} \subset \Xi_{1}, \Xi_{2}$ is called primitive in $\Xi_{1}$ if, for every $\xi \in \Xi_{1}$ such that $p \xi \in \Xi_{2}, p \xi \neq 0$, there exists an element $\xi_{1} \in \Xi_{1}$ for which $p \xi_{1}=0$, and $\xi+\xi_{1} \in \Xi_{2}$.

DEFINITION 4.2. If $\Xi_{1}$ is a $Q$-module, then the index of an element $\xi \in \Xi_{1}$, written $i\left(\xi, \Xi_{1}\right)$, is defined as follows:

(1) For $\xi \in \Xi_{1} \wedge H$, $\xi$ minimally annihilated by $\prod_{j=1}^{k} p_{j}^{t_{j}}, p_{j}$ a prime of $Q$ and $t_{j} \geqq 1$ for $j=1,2, \cdots, k, i\left(\xi, \Xi_{1}\right)$ is the maximum integer $s$ for which there exists an element $\eta \in \Xi_{1}$ such that

$$
\prod_{j=1}^{k} p_{j}^{t_{j}-1} \xi=\prod_{j=1}^{k} p_{j}^{s_{j}+t_{j}-1} \eta, \quad \sum_{j=1}^{k} s_{j}=s .
$$

(2) For $\xi$ regular, $i\left(\xi, \Xi_{1}\right)$ is the maximum integer $s$ for which there exist primes $q_{1}, q_{2}, \cdots, q_{n}$ in $Q$ and $\eta \in \Xi_{1}$ such that

$$
\xi \equiv \prod_{j=1}^{k} q_{j}^{* j} \eta \bmod \Xi_{1} \wedge H, \quad \sum_{j=1}^{k} s_{j}=s .
$$

If in either case this maximum does not exist, $i\left(\xi, \Xi_{1}\right)=\infty: i\left(0, \Xi_{1}\right)=0$.

If $\Xi_{1}$ has non-regular component $H_{1}$, and $\eta \in H_{1}$, then obviously $i\left(\eta, H_{1}\right)$ $=i\left(\eta, \Xi_{1}\right)$. Also, for $\Xi_{2} \subset \Xi_{1}, \eta \in \Xi_{2}, i\left(\eta, \Xi_{2}\right) \leqq i\left(\eta, \Xi_{1}\right)$.

Theorem 4.3. Let $\Xi_{1}$ be a $Q$-module, and $\Xi_{1} \wedge H=H_{1}+H_{2}+\cdots, H_{j}$ $=\Xi_{1} \wedge H_{p_{j}}$. Then for any $\eta \in \Xi_{1} \wedge H$ so that $\eta=\eta_{1}+\eta_{2}+\cdots+\eta_{n}, \eta_{j} \in H_{j}$, 


$$
i\left(\eta, \Xi_{1}\right)=\sum_{j=1}^{n} i\left(\eta_{j}, \Xi_{1}\right) .
$$

To prove this, we first refer back to Theorem 1.4. If $\eta$ has minimum annihilator $\prod_{i=1}^{n} p_{i}^{t_{i}}$, then there exist elements $a_{j} \in Q$ such that

$$
\sum_{j=1}^{n} a_{j} \prod_{i=1, i \neq j}^{n} p_{i}^{t_{i}}=1, \quad \eta_{j}=a_{j} \prod_{i=1, i \neq j}^{n} p_{i}^{t_{i}} \eta
$$

If $i\left(\eta, \Xi_{1}\right)=s<\infty$, then there exists an $\alpha \in \Xi_{1}$ such that

$$
\prod_{i=1}^{n} p_{i}^{t_{i}-1} \eta=\prod_{i=1}^{n} p_{i}^{t_{i}+s_{i}-1} \alpha, \quad \sum_{j=1}^{n} s_{j}=s .
$$

From (1) and (2) we derive

$$
p_{j}^{t_{j}-1} \eta_{j}=p_{j}^{t_{j}+s_{j}-1}\left(a_{j} \prod_{i=1, i \neq j}^{n} p_{i}^{t_{i}+s_{i}} \alpha\right)
$$

so that $i\left(\eta_{j}, \Xi_{1}\right) \geqq s_{j}$. Thus

$$
i\left(\eta, \Xi_{1}\right) \leqq \sum_{j=1}^{n} i\left(\eta_{j}, \Xi_{1}\right) .
$$

On the other hand, let $i\left(\eta_{j}, \Xi_{1}\right)=r_{j}$, so that

$$
p_{j}^{t_{j}} \eta_{j}=p_{j}^{r_{j}+t_{j}-1} \alpha_{j}, \quad j=1,2, \cdots, n .
$$

From (1), this implies

$$
a \prod_{i=1}^{n} p_{i}^{t_{i}-1} \eta=\sum_{j=1}^{n} p_{j}^{r_{j}+t_{j}-1} \alpha_{j}, \quad a=\sum_{j=1}^{n} a_{j} \prod_{i=1, i \neq j}^{n} p_{i} .
$$

As $\left(a, p_{j}\right)=1, j=1,2, \cdots, n, a \bar{a}+\not p_{1} p_{2} \cdots p_{n}=1$ for some $\bar{a}, \bar{p} \in Q$. If we define $c_{j}$ and $d_{j}, j=1,2, \cdots, n$, as solutions of the equations

$$
c_{j} \prod_{i=1, i \neq j}^{n} p_{i}^{r_{i}+t_{i}}+d_{j} p_{j}^{t_{j}}=1, \quad j=1,2, \cdots, n
$$

then for $\beta_{j}=c_{j} \alpha_{j}, j=1,2, \cdots, n$ it follows that

$$
\alpha_{j}=\prod_{i=1, i \neq j}^{n} p_{i}^{r_{i}+t_{i}} \beta_{j}, \quad j=1,2, \cdots, n .
$$

A substitution of this in (3) yields

$$
\prod_{i=1}^{n} p_{i}^{t_{i}-1} \eta=\prod_{i=1}^{n} p_{i}^{r_{i}+t_{i}-1}\left(\bar{a} \sum_{j=1}^{n} \prod_{i=1, i \neq j}^{n} p_{i} \alpha_{j}\right),
$$


so that

$$
i\left(\eta, \Xi_{1}\right) \geqq \sum_{j=1}^{n} i\left(\eta_{j}, \Xi_{1}\right) .
$$

In case $i\left(\eta, \Xi_{1}\right)=\infty$, it will be possible to find an $\alpha$ in (2) for which $s$ exceeds any given number. Thus it must be possible to find an $\alpha$ for which one of the $s_{j}$ exceeds any given number. This implies $i\left(\eta_{j}, \Xi_{1}\right)=\infty$ for some value $j$. Thus the theorem is seen to hold in all cases.

If $\eta \in H_{p}, p$ a prime, and $\eta$ minimally annihilated by $p^{t}$, then for any $a \in Q, a=p^{r} a_{1}$ with $\left(a_{1}, p\right)=1$,

$$
i\left(a \eta, H_{p}\right)=i\left(\eta, H_{p}\right)+r
$$

in case $r<t$. This immediately leads to the following

Corollary 4.4. For $\eta \in \Xi_{1} \wedge H, \eta$ minimally annihilated by $\prod_{i=1}^{n} p_{i}^{t_{i}}$, and $a=a_{1} \prod_{i=1}^{m} p_{i}^{r_{i}}, a_{1}$ a unit, $m \geqq n$, and $a \eta \neq 0$,

$$
i\left(a \eta, \Xi_{1}\right)=i\left(\eta, \Xi_{1}\right)+\sum_{j=1, r_{j}<t_{j}}^{n} r_{j} .
$$

For a regular element $\xi$ of $\Xi_{1}$,

$$
i\left(a \xi, \Xi_{1}\right)=i\left(\xi, \Xi_{1}\right)+\sum_{j=1}^{m} r_{j}
$$

The importance of the concepts of primitivity and index in connection with our problem is seen in the next theorem.

THEOREM 4.5. Let the Q-module $\Xi_{2}$ be primitive in the Q-module $\Xi_{1}$, and either $\Xi_{1} \subset H_{p}$ for some prime $p$ or $\Xi_{1}$ be regular. Then, for any element $\xi$ of $\Xi_{1}-\Xi_{2}$ such that $i\left(\xi, \Xi_{1} / \Xi_{2}\right)=0$ and $\xi$ has the same minimum annihilator $\bmod \Xi_{2}$ and $\bmod 0, \Xi_{2}+Q \xi$ is primitive in $\Xi_{1}$.

In the first place, if $\Xi_{1} \subset H_{p}$, then $\xi$ will have some power of $p$, say $p^{t}$, as minimum annihilator. By assumption, $a \xi \equiv 0 \bmod \Xi_{2}$ implies $a \xi=0$, so that the sum $\Xi_{2}+Q \xi$ is supplementary. Let $p^{8}$ be the minimum annihilator $\bmod \left(\boldsymbol{\Xi}_{2}+Q \xi\right)$ of an element $\eta \in \Xi_{1}$, with $p^{8} \eta \neq 0 \bmod \Xi_{2}$. Thus $p^{8} \eta \equiv a \xi \bmod \boldsymbol{\Xi}_{2}$ for some a $\in Q$, so that $i\left(a \xi, \Xi_{1} / \Xi_{2}\right) \geqq s$. If $a=b p^{r},(b, p)=1$, then $i\left(a \xi, \Xi_{1} / \Xi_{2}\right)=r$ by Corollary 4.4. Thus $s \leqq r$, so that $p^{s}\left(\eta-p^{r-s} b \xi\right) \equiv 0 \bmod \Xi_{2}$. As $\Xi_{2}$ is primitive in $\Xi_{1}$, there must exist an element $\xi_{1}$ of $\Xi_{1}$ for which $p^{8} \xi_{1}=0$ and $\eta-p^{r-s} b \xi+\xi_{1} \in \Xi_{2}$. This last can be written $\eta+\xi_{1} \in \Xi_{2}+Q \xi$, which establishes the primitivity of $\Xi_{2}+Q \xi$ in $\Xi_{1}$.

In case $\Xi_{1}$ is regular, let $\eta$ be a nonzero element of $\Xi_{1}$ for which $a \eta \equiv 0 \bmod \Xi_{2}+Q \xi, a \neq 0$. This means that there exists an element $b \in Q$ such that $a \eta \equiv b \xi \bmod \Xi_{2}$. If $(a, b)=d=r a+s b, b=d b_{1}, a=d a_{1}$, then let $\xi_{2}=r \xi+s \eta$. We then see that $a \xi_{2} \equiv d \xi \bmod \Xi_{2}$, or, because of the primitivity of $\Xi_{2}$ in $\Xi_{1}$, 
$\xi \equiv a_{1} \xi_{2} \bmod \Xi_{2}$. As $i\left(\xi, \Xi_{1} / \Xi_{2}\right)=0, a_{1}$ must be a unit of $Q$ so that $a \mid b$ and $\eta \equiv a_{1}^{-1} b_{1} \xi \bmod \Xi_{2}$. Thus $\eta \in \Xi_{2}+Q \xi$ and the theorem is established.

Now suppose $\Xi_{1}$ is a $Q$-module, and $\Xi_{1} / H_{1}$ has a proper $Q$-basis, $H_{1}$ being the set of all non-regular elements of $\Xi_{1}$. Let this basis be $\left(\xi_{1}+H_{1}, \xi_{2}+H_{1}, \cdots\right)$. If $\sum_{i=1}^{n} a_{i} \xi_{i} \equiv 0 \bmod H_{1}$, then $a_{i} \xi_{i} \equiv 0 \bmod H_{1}, i=1,2, \cdots, n$, so that $\xi_{i}=0$, $i=1,2, \cdots, n$. Thus

$$
\Xi_{1}=H_{1}+Q \xi_{1}+Q \xi_{2}+\cdots,
$$

and the following theorem is seen to hold in view of Theorem 1.4.

THEOREM 4.6. If $\Xi_{1}$ is a $Q$-module, then $\Xi_{1}$ has a proper $Q$-basis if and only if

(1) each nonzero $\Xi_{1} \wedge H_{p_{i}}$ has a proper $Q$-basis, $i=1,2, \cdots$, and

(2) $\Xi_{1} / \Xi_{1} \wedge H$ has a proper $Q$-basis in case $\Xi_{1} \neq \Xi_{1} \wedge H$.

THEOREM 4.7. If $\Xi_{1}$ has a proper $Q$-basis, then all the elements of $\Xi_{1}$ have finite index in $\boldsymbol{\Xi}_{1}$.

To establish this, suppose first that $i\left(\eta, \Xi_{1}\right)=\infty$ for an element $\eta \in H \wedge \Xi_{1}$. From Theorem 4.3, we see that there must exist an integer $r$ and an element $\alpha \in H_{p_{r}} \wedge \Xi_{1}$ such that $i\left(\alpha, H_{p_{r}} \wedge \Xi_{1}\right)=\infty$. Let $H_{p_{r}} \wedge \Xi_{1}=Q \eta_{1}+Q \eta_{2}+\cdots$, and $\alpha=\sum_{i=1}^{n} a_{i} \eta_{i}$. By assumption, if $\alpha$ has minimum annihilator $p_{r}^{z}$, there exist elements $\alpha_{i} \in H_{p_{r}} \wedge \Xi_{1}$ and in tegers $t_{1}<t_{2}<\cdots$ such that

$$
p_{r}^{t-1} \alpha=p_{r}^{t_{j}} \alpha_{j}, \quad j=1,2, \cdots .
$$

If $\alpha_{j}=\sum_{i=1}^{s_{j}} a_{j i} \eta_{i}$, then

$$
p_{r}^{t-1} a_{i} \eta_{i}=p_{r}^{t_{j}} a_{j i} \eta_{i}, \quad i=1,2, \cdots, n ; j=1,2, \cdots .
$$

However, each $\eta_{i}$ is annihilated by some power of $p_{r}$ : therefore this last equation implies that $p_{r}^{t-1} \alpha=0$, which contradicts our assumptions. Thus no nonregular element of $\Xi_{1}$ can have infinite index.

Now assume $i\left(\xi, \Xi_{1}\right)=\infty$ for a regular element $\xi$ of $\Xi_{1}$. Let a proper $Q$-basis of $\Xi_{1}$ be $\left(\eta_{1}, \eta_{2}, \cdots, \xi_{1}, \xi_{2}, \cdots\right)$, with the $\eta_{i} \in H$ and the $\xi_{i}$ regular. Thus $p \xi=p \sum_{i=1}^{n} a_{i} \xi_{i}, p \neq 0$. By assumption, there must exist nonzero elements $q_{j}, r_{j} \in Q$ and $\beta_{j} \in \Xi_{1}$ such that $r_{j} \xi=r_{j} q_{j} \beta_{j}, j=1,2, \cdots$, with the number of prime factors of $q_{j}$ increasing with $j$. If

then

$$
r_{j} \beta_{j}=r_{j} \sum_{i=1}^{s_{j}} a_{j i} \xi_{i}, \quad j=1,2, \cdots,
$$

$$
\operatorname{pr}_{j} \sum_{i=1}^{n} a_{i} \xi_{i}=p q_{j}, r_{j} \sum_{i=1}^{s_{j}} a_{j i} \xi_{i}, \quad \quad i=1,2, \ldots
$$

Thus 


$$
p r_{j}\left(a_{i}-q_{j} a_{j i}\right) \xi_{i}=0, \quad i=1,2, \cdots, n ; j=1,2, \cdots,
$$

so that

$$
a_{i}=q_{j} a_{j i}, \quad i=1,2, \cdots, n ; j=1,2, \cdots .
$$

If $a_{i}$ has $t_{i}$ prime factors, then $j$ can be taken so large that $q_{j}$ has more than $t_{i}$ prime factors, $i=1,2, \cdots, n$. This is impossible, so that no regular element of $\Xi_{1}$ can have infinite index. This establishes the theorem.

THEOREM 4.8. Let $H_{1}$ be a $Q$-module with the property that every element of $H_{1}$ is annihilated by some power of the prime $p$. Then $H_{1}$ has a proper $Q$-basis if and only if for every primitive set $H_{2} \subset H_{1}, H_{2}$ having a finite Q-basis, all the elements of $H_{1} / H_{2}$ have finite index.

To prove the necessity of this condition, let $H_{1}=Q \eta_{1}+Q \eta_{2}+\cdots$ and define $H_{1}^{k}=Q \eta_{1}+Q \eta_{2}+\cdots+Q \eta_{k}$. For any $Q$-submodule $H_{2}$ of $H_{1}$ which possesses a finite $Q$-basis, there exists an integer $n$ such that $H_{2} \subset H_{1}^{n}$. Thus

$$
H_{1}^{n} / H_{2}=Q \alpha_{1}+Q \alpha_{2}+\cdots+Q \alpha_{m}
$$

by Theorem 3.4. This shows that

$$
H_{1} / H_{2}=Q \alpha_{1}+Q \alpha_{2}+\cdots+Q \alpha_{m}+Q \eta_{n+1}+Q \eta_{n+2}+\cdots,
$$

and thus all the elements of $H_{1} / H_{2}$ have finite index by Theorem 4.7.

To establish the converse, let $\left(\eta_{1}, \eta_{2}, \cdots\right)$ be any $Q$-basis of $H_{1}$. Assume that $\Gamma^{m}$ is a $Q$-module primitive in $H_{1}, \Gamma^{m}$ has a proper $Q$-basis $\left(\alpha_{1}, \alpha_{2}, \cdots, \alpha_{m}\right)$, and $i\left(\alpha_{j}, H_{1} / \Gamma^{j-1}\right)=0, j=1,2, \cdots, m$ with $\Gamma^{0}=0$. Also assume

$$
Q\left[\eta_{1}, \eta_{2}, \cdots, \eta_{k-1}\right] \subset \Gamma^{m} \subset H_{1} .
$$

If $i\left(\eta_{k}, H_{1} / \Gamma^{m}\right)=r>0$, with $p^{i_{1}}$ the minimum annihilator of $\eta_{k} \bmod \Gamma^{m}$, then there exists an $\alpha_{m+1} \in H_{1}$ such that

$$
p^{t_{1}-1} \eta_{k} \equiv p^{r+t_{1}-1} \alpha_{m+1} \bmod \Gamma^{m}, \quad p^{r+t_{1}} \alpha_{m+1}=0 .
$$

Thus $i\left(\alpha_{m+1}, H_{1} / \Gamma^{m}\right)=0$, and by Theorem $4.5, \Gamma^{m+1}=\Gamma^{m}+Q \alpha_{m+1}$ is primitive in $H_{1}$. If $p^{t_{2}}$ is the minimum annihilator of $\eta_{k} \bmod \Gamma^{m+1}$, then $t_{2}<t_{1}$. As above, there exists an $\alpha_{m+2} \in H_{1}, i\left(\alpha_{m+2}, H_{1} / \Gamma^{m+1}\right)=0$, and $\Gamma^{m+1}+Q \alpha_{m+2}$ is primitive in $H_{1}$. There will exist an integer $n$ and elements $\alpha_{m+3}, \alpha_{m+4}, \cdots, \alpha_{m+n} \in H_{1}$, with $\Gamma^{m+n}=Q \alpha_{1}+Q \alpha_{2}+\cdots+Q \alpha_{m+n}, i\left(\alpha_{j}, H_{1} / \Gamma^{j-1}\right)=0, j=1,2, \cdots, m+n$, and $\Gamma^{m+n}$ primitive in $H_{1}$, such that

$$
Q\left[\eta_{1}, \eta_{2}, \cdots, \eta_{k}\right] \subset \Gamma^{m+n} \subset H_{1} .
$$

Thus, by induction, there exist elements $\alpha_{1}, \alpha_{2}, \cdots \in H_{1}$ such that

$$
H_{1}=Q \alpha_{1}+Q \alpha_{2}+\cdots \text {. }
$$

COROLlary 4.9. If the $Q$-module $\Xi_{1}$ is annihilated by a nonzero element $h \in Q$, then $\Xi_{1}$ has a proper $Q$-basis. 
If $h=\prod_{i=1}^{n} p_{i}^{t_{i}}$, then

$$
\Xi_{1}=H_{1}+H_{2}+\cdots+H_{n}, \quad p_{i}^{t_{i}} H_{i}=0, \quad i=1,2, \cdots, n .
$$

Thus for any $Q$-module $H_{i}^{\prime} \subset H_{i}$, all the elements of $H_{i} / H_{i}^{\prime}$ have finite index, and the corollary follows from Theorem 4.8.

THEOREM 4.10. If $\Xi_{1} \neq \Xi_{1} \wedge H$, then $\Xi_{1} / \Xi_{1} \wedge H$ has a proper $Q$-basis if and only if, for every regular $Q$-module $K \subset \Xi_{1}$ such that $K$ has a finite $Q$-basis and $H \wedge \Xi_{1}+K$ is primitive in $\Xi_{1}$, all elements of $\Xi_{1} / \Xi_{1} \wedge H+K$ have finite index.

The proof of this theorem is similar to that of the last theorem. If $\Xi_{1} \wedge H=H_{1}$, and $\Xi_{1}$ has a proper $Q$-basis, then $\Xi_{1}=H_{1}+\Omega_{1}, \Omega_{1}$ a regular $Q$-module. Let $\left(\xi_{1}, \xi_{2}, \cdots\right)$ be a proper $Q$-basis for $\Omega_{1}$. For any regular $Q$-module $K$ which possesses a finite $Q$-basis there exists an integer $n$ such that

$$
H_{1}+K \subset H_{1}+Q \xi_{1}+Q \xi_{2}+\cdots+Q \xi_{n} .
$$

As in Theorem 4.8, we see that $\Xi_{1} / H_{1}+K$ has a proper $Q$-basis, and thus all its elements have finite index.

Conversely, let $\Xi_{1}=Q\left[\beta_{1}, \beta_{2}, \cdots\right] \vee H_{1}$, all $\beta_{i}$ being regular. Assume that we have found regular elements $\xi_{1}, \xi_{2}, \cdots, \xi_{m}$ in $\Xi_{1}$ which are $Q$-linearly independent such that, if $\Gamma^{n}=Q \xi_{1}+Q \xi_{2}+\cdots+Q \xi_{n}+H_{1}$,

$$
i\left(\xi_{j}, \Xi_{1} / \Gamma^{j-1}\right)=0, \quad j=1,2, \cdots, m,
$$

and

$$
Q\left[\beta_{1}, \beta_{2}, \cdots, \beta_{k-1}\right] \vee H_{1} \subset \Gamma^{m} \subset \Xi_{1} .
$$

Thus $\Gamma^{m}$ is primitive in $\Xi_{1}$. If $i\left(\beta_{k}, \Xi_{1} / \Gamma^{m}\right) \neq 0$, then there exists an element $\xi_{m+1} \in \Xi_{1}$ such that

$$
\beta_{k} \equiv a \xi_{m+1} \bmod \Gamma^{m}, \quad i\left(\xi_{m+1}, \Xi_{1} / \Gamma^{m}\right)=0 .
$$

Thus

$$
Q\left[\beta_{1}, \beta_{2}, \cdots, \beta_{k}\right] \vee H_{1} \subset \Gamma^{m+1} \subset \Xi_{1},
$$

and the theorem follows by induction.

It is always desirable to have the important properties of any set carry over to "admissible" subsets. In this case, the property of a module having a proper basis should carry over to submodules. This was seen to be the case for $P$-modules in Theorem 1.1. That such is also the case for $Q$-modules is demonstrated in the next theorem.

TheOREM 4.11. If $\Xi_{1}$ has a proper $Q$-basis, then any $Q$-submodule $\Xi_{2}$ of $\Xi_{1}$ also has a proper Q-basis. 
This is a consequence of Theorems 4.8 and 4.10. Let $H_{j}=H_{p_{1}} \wedge \Xi_{j}, j=1,2$, and assume $H_{2} \neq 0$. Then for any primitive set $H_{3} \subset H_{2}, H_{3}$ having a finite $Q$-basis, and any element $\eta \in H_{2}, i\left(\eta, H_{2} / H_{3}\right) \leqq i\left(\eta, H_{1} / H_{3}\right)$. Now, even though $H_{3}$ need not be primitive in $H_{1}, i\left(\eta, H_{1} / H_{3}\right)<\infty$. Thus $i\left(\eta, H_{2} / H_{3}\right)<\infty$ so that $H_{2}$ has a proper $Q$-basis. A similar argument also shows that $\Xi_{2} / H \wedge \Xi_{2}$ has a proper $Q$-basis, so that $\Xi_{2}$ has a proper $Q$-basis by Theorem 4.6.

5. The modular case. The statement that $Q$ is an operator domain of $\Xi$ carries with it the assumption that $a \xi=b \xi$ for all $\xi \in \Xi$ implies $a=b$. We will now consider the case in which $a \xi=b \xi$ for all $\xi \in \Xi$ implies $a \equiv b \bmod h, h$ not a unit. This is equivalent to the statement that $Q /(h)$ is an operator domain of $\Xi$. Let $R=Q /(h)$ and $h=\prod_{i=1}^{t} p_{i}^{t_{i}}, p_{i}$ primes of $Q$ and $t_{i} \geqq 1, i=1,2, \cdots, t$. As above, $P \subset R$, and the Fundamental Axiom will still be assumed.

If $h$ is a prime, then $R$ is a field and $\Xi$ is a regular $R$-module. Otherwise, it is apparent that the minimum annihilators of the non-regular elements of $\Xi$ are divisors of $h$. In this case, if $\xi$ is regular, $p_{1} \xi$ is non-regular, so that in view of Theorem 1.4 we have

$$
\Xi=H_{p_{1}}+H_{p_{2}}+\cdots+H_{p_{i}} \text {. }
$$

\section{TheOREM 5.1. The set $\Xi$ has a proper $R$-basis.}

If $R$ is a field, this follows from Theorem 1.1. Otherwise, the proof will be to show that $H_{p_{1}}$ has a proper $R$-basis, which will lead to a proper $R$-basis for $\Xi$ in view of (1). For simplification, let $H_{p_{1}}=H, t_{1}=n, p_{1}=p$, and define $H_{i}$ to be the maximum submodule of $H$ annihilated by $p^{i}, 0 \leqq i \leqq n, H_{0}=0$. Thus $H_{0} \subset H_{1} \subset \cdots \subset H_{n}=H$.

The $R$-module $H_{m} / H_{m-1}, 0<m \leqq n$, is annihilated by $p$. Let $\left(\xi_{1}, \xi_{2}, \cdots\right)\left(^{4}\right)$ be a $P$-basis for this $R$-module. Now discard all $\xi_{i} \equiv 0 \bmod R\left[\xi_{1}, \xi_{2}, \cdots, \xi_{i-1}\right]$ $\bigvee H_{m-1}$. If we label the remaining set $\left(\bar{\xi}_{1}, \bar{\xi}_{2}, \cdots\right)$, then this set is a proper $R$-basis for $H_{m} / H_{m-1}$ and is $R$-linearly independent in $H_{m}$. For, if

$$
p^{m-1} \sum_{i=1}^{k} a_{i} \bar{\xi}_{i}=0, \quad a_{k} \not \equiv 0 \bmod p,
$$

then $a_{k} \xi_{k} \equiv 0 \bmod R\left[\bar{\xi}_{1}, \bar{\xi}_{2}, \cdots, \bar{\xi}_{k-1}\right] \bigvee H_{m-1}$. However, as $a_{k}$ has an inverse $\bmod p$, this implies that $\bar{\xi}_{k} \equiv 0 \bmod R\left[\bar{\xi}_{1}, \bar{\xi}_{2}, \cdots, \bar{\xi}_{k-1}\right] \bigvee H_{m-1}$, which is impossible in view of the method of selection of $\bar{\xi}_{k}$.

Assume that $H / H_{m+1}$ has a proper $R$-basis $\left(\alpha_{1}, \alpha_{2}, \cdots\right)$ for some integer $m, 0 \leqq m<n-1$, this basis being a $R$-linearly independent set in $H$. Let $\left(\beta_{1}, \beta_{2}, \cdots\right)$ be a proper $R$-basis for $H_{m+1} / H_{m}$. Now discard all $\beta_{j}$ such that

$$
\beta_{j} \equiv 0 \bmod R\left[\alpha_{1}, \alpha_{2}, \cdots\right] \vee H_{m} \vee R\left[\beta_{1}, \beta_{2}, \cdots, \beta_{j-1}\right] .
$$

Denote those remaining by $\left(\gamma_{1}, \gamma_{2}, \cdots\right)$. From the way that the set

(4) The basis is in reality $\left(\xi_{1}+H_{m-1}, \xi_{2}+H_{m-1}, \cdots\right)$-see the last paragraph of $\S 1$. 
$\left(\alpha_{1}, \alpha_{2}, \cdots, \gamma_{1}, \gamma_{2}, \cdots\right)$ was chosen, it can be seen that it is a proper $R$-basis for $H / H_{m}$. To show that this set is $R$-linearly independent in $H$, suppose

$$
p^{m+1} \sum_{i=1}^{r} a_{i} \alpha_{i}=p^{m} \sum_{i=1}^{k} c_{i} \gamma_{i},
$$

where $c_{k}$ is not congruent to zero $\bmod p$. Then $c_{k}$ has an inverse $\bmod p$, so that

$$
\gamma_{k} \equiv 0 \bmod R\left[\alpha_{1}, \alpha_{2}, \cdots\right] \vee H_{m} \vee R\left[\gamma_{1}, \gamma_{2}, \cdots, \gamma_{k-1}\right]
$$

This contradicts the method of selection of $\gamma_{k}$. Thus, by induction, $H=H / H_{0}$ has a proper $R$-basis.

6. Applications to infinite matrices. The ordered set $(1,2, \cdots, n, \cdots)$ of type $\omega$ will be denoted by $\Delta$. In what is to follow, $x$ will denote a commutative indeterminate over $P$, and $P[x]$ will denote the polynomial domain in $x$ over $P$. The $P$-module to be used as the $\Xi$ above is defined as follows:

Definition 6.1. $\Xi$ is the $P$-module composed of all vectors $\left(a_{i} ; i \in \Delta\right)$ with elements in $P$ such that only a finite number of the elements in each vector are different from zero. Addition is ordinary vector addition.

The vector $\left(a_{i} ; i \in \Delta\right)$ with $a_{j}=1, a_{i}=0$ for $i \neq j$ is denoted by $\delta_{j}$. The set of vectors $\left(\delta_{1}, \delta_{2}, \cdots\right)$ is a proper $P$-basis for $\Xi$, and thus the Fundamental Axiom is satisfied.

The total matric algebra of order $n^{2}$ over $P$ is denoted by $\mathfrak{M}_{n}$. If $A$ is an element of $\mathfrak{M}_{n}$, and $\eta$ is an element of the total vector space $\mathfrak{B}_{n}$ of order $n$ over $P$, then $A \eta$ (considering $\eta$ as an $n \times 1$ matrix) is again an element of $\mathfrak{B}_{n}$. Thus $A$ is an operator of $\mathfrak{B}_{n}$, and $\mathfrak{M}_{n}$ is the total operator domain of $\mathfrak{B}_{n}$.

A total operator domain exists for $\mathbb{\Xi}$, and is equivalent to the ring $\mathfrak{M}_{\omega}$ below.

Definition 6.2. $\mathfrak{M}_{\omega}$ is the set of all matrices $\left(a_{r s} ; r, s \in \Delta\right)$ over $P$ with the property that the vectors $\left(a_{r s} ; r \in \Delta\right)$ are in $\Xi$ for all $s \in \Delta$.

For $A, B \in \mathfrak{M}_{\omega}, A=\left(a_{r s} ; r, s \in \Delta\right), B=\left(b_{r s} ; r, s \in \Delta\right)$, the sum and product of these are defined as usual-that is,

$$
A B=\left(\sum_{i} a_{r i} b_{i s} ; r, s \in \Delta\right), \quad A+B=\left(a_{r s}+b_{r s} ; r, s \in \Delta\right) .
$$

Under these operations of (finite) sum and product, $\mathfrak{M}_{\omega}$ is a ring. The element $I=\left(a_{r s} ; r, s \in \Delta\right)$, with $a_{r s}=0$ for $r \neq s$, and $a_{r r}=1$ is the unit element of $\mathfrak{M}_{\omega}$. The notation above will be simplified by omitting the range of the indices when there is no chance of ambiguity. The notation $\sum_{i}$ means that the summation is taken over $\Delta$.

The matrices of $\mathfrak{M}_{\omega}$ are left operators of $\Xi$ under the following definition: 
for $A \in \mathfrak{M}_{\omega}, \eta \in \Xi$ with $A=\left(a_{r s}\right), \eta=\left(c_{i}\right)$,

$$
A \eta=\left(\sum_{j} a_{i j} c_{j} ; i \in \Delta\right) .
$$

One can think of $\mathfrak{M}_{\omega}$ also as a vector space with elements from $\Xi$. If $A \in \mathfrak{M}_{\omega}, A=\left(a_{r s}\right)$, then $A=\left(\alpha_{i} ; i \in \Delta\right)$, where $\alpha_{j}=\left(a_{i j} ; i \in \Delta\right)$.

An element $A$ of $\mathfrak{M}_{\omega}$ is regular in case $A$ possesses an inverse in $\mathfrak{M}_{\omega}$. The ring $\mathfrak{M}_{\omega}$ possesses elements which are semi-regular-that is, elements which have a right inverse but not a left, or vice versa. Such an element is $N$ defined below (Definition 6.10). The element $A$ is algebraic in case there exists a nonzero $m(x) \in P[x]$ such that $m(A)=0$. Thus, if $A$ is algebraic, there exists a polynomial $h(x)$ of minimal degree, called the minimal polynomial of $A$, such that $h(A)=0$.

For any non-algebraic element $A$ of $\mathfrak{M}_{\omega}$, the polynomial domain $P[A]$ is a principal ideal ring. In what is to follow, these principal ideal rings correspond to the ring $Q$ used above. In case $A$ is algebraic with minimal polynomial $h(x)$, then $P[A]$ is isomorphic to the ring $P[x] / h(x)$, and the theory of $\$ 5$ is applicable.

THEOREM 6.3. If $\left(\xi_{1}, \xi_{2}, \cdots\right)$ is a proper P-basis for $\Xi$, then $C=\left(\xi_{i} ; i \in \Delta\right)$ is a regular element of $\mathfrak{M}_{\omega}$.

To prove this, we see that there must exist $a_{i j} \in P$ such that $\sum_{i} a_{i j} \xi_{i}=\delta_{j}$. Let $A=\left(a_{r s}\right)$; then $C A=I$, so that $A$ is a right inverse of $C$. Now $C A C-C=0$, so $C(A C-I)=0$. If $(A C-I)=\left(b_{r s}\right) \neq 0$, there exists an integer $n$ such that $\eta=\left(b_{r n} ; r \in \Delta\right) \neq 0$. Then $C \eta=\sum_{i} b_{i n} \xi_{i}=0$, which contradicts the hypothesis that $\left(\xi_{1}, \xi_{2}, \cdots\right)$ is a proper $P$-basis for $\Xi$. Thus $A C=I$, and $C$ is regular.

Definition 6.4. An element $A$ of $\mathfrak{M}_{\omega}$ is said to be reducible if and only if $\Xi$ has a proper $P[A]$-basis.

The definition of direct sums of finite matrices (see [4, p. 237]) can be carried over to $\mathfrak{M}_{\omega}$. Thus an element $A$ of $\mathfrak{M}_{\omega}$ is the direct sum ( $\dot{+}$ ) of the elements $A_{1}$ of $\mathfrak{M}_{n}$ and $A_{2}$ of $\mathfrak{M}_{\omega}, A=A_{1}+A_{2}$, if and only if, for $A=\left(a_{r s}\right)$, $A_{1}=\left(a_{1 r s}\right), A_{2}=\left(a_{2 r s}\right), a_{r s}=a_{1 r s}$ for $r, s \leqq n, a_{r+n, s+n}=a_{2 r s}$ for $r, s \in \Delta$ and otherwise $a_{r s}=0$. By iteration, the direct sum of an infinite number of finite matrices can be defined.

Definition 6.5. An element $A$ of $\mathfrak{M}_{\omega}$ is in reduced form in case $A$ is the direct sum of finite matrices.

As in the finite case, two elements $A$ and $B$ of $\mathfrak{M}_{\omega}$ are similar in case there exists a regular element $T$ of $\mathfrak{M}_{\omega}$ such that $B=T^{-1} A T$.

Theorem 6.6. If $A$ is reducible, then any element $B$ similar to $A$ is also reducible. 
Let $\Xi=P[A] \xi_{1}+P[A] \xi_{2}+\cdots, B=T^{-1} A T$. Now define $\eta_{n}=T^{-1} \xi_{n}$, $n=1,2, \cdots$. Then $\Xi=P[B] \eta_{1}+P[B] \eta_{2}+\cdots$, so that $B$ is reducible.

Theorem 6.7. If $A$ is in reduced form, then $A$ is reducible.

That this is true is a consequence of the fact that $\mathfrak{B}_{n}$ has a proper $P[B]$-basis for any $B \in \mathfrak{M}_{n}$.

THEOREM 6.8. If $A$ is reducible and all the elements of $\Xi$ are non-regular with respect to $P[A]$, then $A$ is similar to an element $B$ in reduced form.

Let $\Xi=P[A] \xi_{1}+P[A] \xi_{2}+\cdots$, with $h_{i}(A)$ the minimum annihilator of $\xi_{i}$. If $t_{i}$ is the degree of $h_{i}(x)$, then

Now define

$$
h_{i}(x)=x^{t_{i}}+\sum_{j=1}^{t_{i}-1} a_{i j} x^{j}
$$

$$
T=\left(\xi_{1}, A \xi_{1}, \cdots, A^{t_{1}-1} \xi_{1}, \xi_{2}, A \xi_{2}, \cdots, A^{t_{2}-1} \xi_{2}, \cdots\right),
$$

so that $T \in \mathfrak{M}_{\omega}$. By Theorem $6.3, T$ is regular in $\mathfrak{M}_{\omega}$. If $A_{i}$ is the companion matrix (5) of $h_{i}(x)$, so that $A_{i} \in \mathfrak{M}_{t_{i}}$, then

$$
T^{-1} A T=A_{1}+A_{2}+\cdots,
$$

and the theorem is established.

This theorem does not include the important case of algebraic matrices. However, in view of Theorem 5.1, a similar proof leads to

TheOREM 6.9. If $A$ is algebraic with minimal polynomial $m(x)$, then $A$ is similar to an element $B$ in reduced form. If $B=B_{1} \dot{+} B_{2} \dot{+} \cdots$, then each $B_{i}$ is the companion matrix of some divisor of $m(x)$.

Definition 6.10. The elements $N$ and $N^{\omega}$ of $\mathfrak{M}_{\omega}$ are defined as follows:

$$
\begin{aligned}
N & =\left(\delta_{2}, \delta_{3}, \cdots\right), \quad N^{0}=0, \\
N^{\omega} & =\left(N \delta_{1}, N^{2} \delta_{2}, N^{2} \delta_{3}, N^{3} \delta_{4}, N^{3} \delta_{5}, N^{3} \delta_{6}, \cdots\right) .
\end{aligned}
$$

That powers of $N$ are of fundamental importance in the study of reducible matrices is seen in the following

TheOREm 6.11. If $A$ is reducible, $A$ not algebraic, and $\Xi$ is a regular $P[A]$-module, then $A$ is similar to some power of $N$.

To prove this, let

$$
\Xi=P[A] \xi_{1}+P[A] \xi_{2}+\cdots+P[A] \xi_{n} .
$$

If $n<\infty$, let

(5) This is the $t_{i} \times t_{i}$ matrix with 1 's directly below the main diagonal, $-a_{i 0},-a_{i 1}, \cdots$, $-a_{i t i-1}$ as the last column, and 0 's elsewhere. 
$T=\left(\xi_{1}, \xi_{2}, \cdots, \xi_{n}, A \xi_{1}, A \xi_{2}, \cdots, A \xi_{n}, A^{2} \xi_{1}, A^{2} \xi_{2}, \cdots, A^{2} \xi_{n}, \cdots\right)$.

Then $T^{-1} A T \doteq N^{n}$. However, if $n=\infty$, let

$$
T=\left(\xi_{1}, A \xi_{1}, \xi_{2}, A^{2} \xi_{1}, A \xi_{2}, \xi_{3}, \cdots\right) \text {. }
$$

Then $T^{-1} A T=N^{\omega}$, and the theorem follows.

The author intends to deal at greater length in a subsequent paper with matrix algebras of different order types. However, a matrix algebra of order type $\omega 2$ will be considered briefly here.

Denote the ordered set $(1,2, \cdots, \omega+1, \omega+2, \cdots)$ of type $\omega 2$ by $\Delta_{2}$. Let $\Xi_{2}$ be the $P$-module composed of all vectors $\left(a_{i} ; i \in \Delta_{2}\right)$ over $P$, with only a finite number of the elements of any vector being different from zero. $\mathfrak{M}_{\omega 2}$ is the ring of all matrices $\left(a_{r s} ; r, s \in \Delta_{2}\right)$ over $P$ with all $\left(a_{r s} ; r \in \Delta_{2}\right)$ in $\Xi_{2}$. Let $\delta_{i}^{\prime}$ be the element of $\Xi_{2}$ which has 1 in the $i$ th place and 0 elsewhere, $i \in \Delta_{\mathbf{2}}$. Then $\left(\delta_{i}^{\prime} ; i \in \Delta_{\mathbf{2}}\right)$ is a proper $P$-basis of $\Xi_{\mathbf{2}}$.

For $A \in \mathfrak{M}_{\omega}, A^{\prime} \in \mathfrak{M}_{\omega 2}$, the correspondence

$$
A \leftrightarrow\left(\begin{array}{ll}
A & 0 \\
0 & 0
\end{array}\right)=A^{\prime}
$$

defines an isomorphism between $\mathfrak{M}_{\omega}$ and a subring of $\mathfrak{M}_{\omega 2}$. (Under a different correspondence, $\mathfrak{M}_{\omega}$ and $\mathfrak{M}_{\omega 2}$ can be shown to be actually isomorphic.) Using the notation of direct sum, $A \leftrightarrow A^{\prime}=A \dot{+} 0$.

If $A$ is reducible so that $\Xi=P[A] \xi_{1}+P[A] \xi_{2}+\cdots$, then define $\xi_{j}^{\prime}=\left(\xi_{j}, a_{\omega+i} ; i \in \Delta\right)$, all $a_{\omega+i}=0:$ thus $\xi_{j}^{\prime} \in \Xi_{2}$. Then

$$
\Xi_{2}=P\left[A^{\prime}\right] \xi_{1}^{\prime}+P\left[A^{\prime}\right] \xi_{2}^{\prime}+\cdots+P\left[A^{\prime}\right] \delta_{\omega+1}^{\prime}+P\left[A^{\prime}\right] \delta_{\omega+2}^{\prime}+\cdots,
$$

so that $A^{\prime}$ is also reducible.

THEOREM 6.12. If $A$ is reducible, then $A^{\prime}$ is similar to $B+N^{k}$ where $B \in \mathfrak{M}_{\omega}$ is in reduced form and $k$ is an integer or $\omega$.

To prove this, let

$$
\Xi_{2}=P\left[A^{\prime}\right] \eta_{1}^{\prime}+P\left[A^{\prime}\right] \eta_{2}^{\prime}+\cdots+P\left[A^{\prime}\right] \xi_{1}^{\prime}+P\left[A^{\prime}\right] \xi_{2}^{\prime}+\cdots+P\left[A^{\prime}\right] \xi_{k}^{\prime} \text {, }
$$

the $\xi_{i}^{\prime}$ being regular and the $\eta_{i}^{\prime}$ non-regular, with $h_{i}(x)$ the minimum annihilator of $\eta_{i}^{\prime}$. The degree of $h_{i}(x)$ is labeled $n_{i}$. Then $T^{\prime} \in \mathfrak{M}_{\omega 2}$ can be chosen (assuming $k$ finite) as

$$
\begin{array}{r}
T^{\prime}=\left(\eta_{1}^{\prime}, A^{\prime} \eta_{1}^{\prime}, \cdots, A^{\prime n_{1}-1} \eta_{1}^{\prime}, \eta_{2}^{\prime}, A^{\prime} \eta_{2}^{\prime}, \cdots, A^{n_{2}-1} \eta_{2}^{\prime}, \cdots, \xi_{1}^{\prime}, \xi_{2}^{\prime}, \cdots, \xi_{k}^{\prime},\right. \\
\left.A^{\prime} \xi_{1}^{\prime}, A^{\prime} \xi_{2}^{\prime}, \cdots, A^{\prime} \xi_{k}^{\prime}, \cdots\right) .
\end{array}
$$

Theorem 6.3 is seen to carry over for $\Xi_{2}$, and thus

$$
T^{-1} A^{\prime} T^{\prime}=B+N^{k},
$$

$B$ reduced. If $k=\infty$, the $\xi_{i}^{\prime}$ in $T^{\prime}$ can be arranged as in Theorem 6.11 . 
From Theorem 2.2, we see that every reducible matrix $A$ of $\mathfrak{M}_{\omega}$ has an associated characteristic matrix. The characteristic matrix of a reducible matrix is of importance in determining the similarity of matrices, as the following theorem shows.

Theorem 6.13. Two reducible matrices $A$ and $B$ of $\mathfrak{M}_{\omega}$ are similar if and only if their characteristic matrices are equal.

To establish this, first assume that $\Xi=P[A] \xi_{1}+P[A] \xi_{2}+\cdots$ and $B=T^{-1} A T$. Then, by Theorem 6.6, $\Xi=P[B] \eta_{1}+P[B] \eta_{2}+\cdots$, where $\eta_{i}=T^{-1} \xi_{i}$. For any $m(x) \in P[x], m(A) \xi_{i}=T m(B) \eta_{i}$, so $\eta_{i}$ is regular with respect to $P[B]$ if $\xi_{i}$ is regular with respect to $P[A]$. Also, if $m(x)$ is the minimum annihilator of $\xi_{i}$ with respect to $P[A], m(x)$ is the minimum annihilator of $\eta_{i}$ with respect to $P[B]$. Thus the characteristic matrix of $A$ equals the characteristic matrix of $B$.

Now assume $A$ and $B$ have the same characteristic matrix. For any proper $P[A]$-basis $\left(\xi_{1}, \xi_{2}, \cdots, \eta_{1}, \eta_{2}, \cdots\right)$ of $\Xi, \xi_{i}$ regular and $\eta_{i}$ minimally annihilated by $m_{i}(x), n_{i}$ being the degree of $m_{i}$, there exists a proper $P[B]$ basis $\left(\bar{\xi}_{1}, \bar{\xi}_{2}, \cdots, \bar{\eta}_{1}, \bar{\eta}_{2}, \cdots\right)$ of $\Xi$ with $\bar{\xi}_{i}$ regular and $\bar{\eta}_{i}$ minimally annihiated by $m_{i}(x)$. There is a 1-1 correspondence $\bar{\xi}_{i} \leftrightarrow \xi_{i}, \bar{\eta}_{i} \leftrightarrow \eta_{i}$ between these two bases. Then let

$T=\left(\xi_{1}, \eta_{1}, A \eta_{1}, \cdots, A^{n_{1}-1} \eta_{1}, A \xi_{1}, \xi_{2}, \eta_{2}, A \eta_{2}, \cdots, A^{n_{2}-1} \eta_{2}, A^{2 \xi_{1}}, A \xi_{2}, \xi_{3}, \cdots\right)$,

and $S$ be the same as $T$ with $A$ replaced throughout by $B$. Then $T^{-1} A T=S^{-1} B S$, so $A$ and $B$ are similar, and the theorem is established.

TheOREM 6.14. If $A$ is reducible and regular, then $A$ is similar to a matrix in reduced form.

If $\Xi$ possesses a regular vector with respect to $P[A]$, then any proper $P[A]$-basis of $\Xi$ must have a regular element $\xi$. Select $T$ as in the proof of Theorem 6.13 with $\xi$ the first vector of $T$. Then $T^{-1} A T$ has its first row (or column) composed of zeros. This implies $T^{-1} A T$ is not regular which means $A$ is not regular. Thus $\Xi$ can have only non-regular elements with respect to $P[A]$, and the theorem follows from Theorem 6.7.

As an example of the reduction of an element of $\mathfrak{M}_{\omega}$ to reduced form, take

$$
A=\left(\delta_{1}+\delta_{3},-\delta_{3}, \delta_{2}-\delta_{3},-\delta_{3}+\delta_{4},-\delta_{3}+\delta_{5},-\delta_{3}+\delta_{6}, \cdots\right) \text {. }
$$

It can be verified that $A$ is algebraic with minimal equation $x^{3}-1=0$. Then $\Xi=P[A] \delta_{1}+P[A]\left(\delta_{1}+\delta_{4}\right)+P[A]\left(\delta_{1}+\delta_{5}\right)+\cdots$, with $A^{3}-1$ the minimum annihilator of $\delta_{1}$ and $A-1$ the minimum annihilator of $\delta_{1}+\delta_{i}, i=4,5, \cdots$. Then $T$ can be chosen as 
so that

$$
\begin{aligned}
T & =\left(\delta_{1}, \delta_{1}+\delta_{3}, \delta_{1}+\delta_{2}, \delta_{1}+\delta_{4}, \delta_{1}+\delta_{5}, \cdots\right) \\
T^{-1} & =\left(\delta_{1},-\delta_{1}+\delta_{3},-\delta_{1}+\delta_{2},-\delta_{1}+\delta_{4},-\delta_{1}+\delta_{5}, \cdots\right)
\end{aligned}
$$

$$
T^{-1} A T=\left(\delta_{2}, \delta_{3}, \delta_{1}, \delta_{4}, \delta_{5}, \delta_{6}, \cdots\right)
$$

\section{BIBLIOGRAPHY}

1. C. Chevalley, L'arithmetique dans les algebres de matrices, Paris, 1936.

2. M. H. Ingraham, $A$ general theory of linear sets, Trans. Amer. Math. Soc. vol. 27 (1925) pp. 163-196.

3. M. H. Ingraham and M. C. Wolf, Relative linear sets and similarity of matrices whose elements belong to a division algebra, ibid. vol. 42 (1937) pp. 16-31.

4. C. C. MacDuffee, Introduction to abstract algebra, New York, 1940.

5. H. Zassenhaus, Lehrbuch der Gruppentheorie, Leipzig, 1937.

UNIVERSITY OF WISCONSIN,

Madison, Wis. 